\title{
Plasma-based longitudinal mutation monitoring as a potential predictor of disease progression in subjects with adenocarcinoma in advanced non-small cell lung cancer
}

John Jiang ${ }^{1}$, Hans-Peter Adams², Maria Lange², Sandra Siemann², Mirjam Feldkamp², Sylvie McNamara², Sebastian Froehler², Stephanie J. Yaung ${ }^{1}$, Lijing Yao ${ }^{1}$, Aarthi Balasubramanyam ${ }^{3}$, Nalin Tikoo ${ }^{3}$, Christine Ju ${ }^{3}$, H. Jost Achenbach ${ }^{4}$, Rainer Krügel ${ }^{5}$ and John F. Palma ${ }^{1 *}$ (D)

\begin{abstract}
Background: Identifying and tracking somatic mutations in cell-free DNA (cFDNA) by next-generation sequencing (NGS) has the potential to transform the clinical management of subjects with advanced non-small cell lung cancer (NSCLC).

Methods: Baseline tumor tissue $(n=47)$ and longitudinal plasma $(n=445)$ were collected from 71 NSCLC subjects treated with chemotherapy. cfDNA was enriched using a targeted-capture NGS kit containing 197 genes. Clinical responses to treatment were determined using RECIST v1.1 and correlations between changes in plasma somatic variant allele frequencies and disease progression were assessed.

Results: Somatic variants were detected in 89.4\% (42/47) of tissue and 91.5\% (407/445) of plasma samples. The most commonly mutated genes in tissue were TP53 (42.6\%), KRAS (25.5\%), and KEAP1 (19.1\%). In some subjects, the allele frequencies of mutations detected in plasma increased 3-5 months prior to disease progression. In other cases, the allele frequencies of detected mutations declined or decreased to undetectable levels, indicating clinical response. Subjects with circulating tumor DNA (ctDNA) levels above background had significantly shorter progression-free survival (median: 5.6 vs 8.9 months, respectively; log-rank $p=0.0183$ ).

Conclusion: Longitudinal monitoring of mutational changes in plasma has the potential to predict disease progression early. The presence of ctDNA mutations during first-line treatment is a risk factor for earlier disease progression in advanced NSCLC.
\end{abstract}

Keywords: Next-generation sequencing (NGS), Cell-free DNA (cfDNA), Circulating tumor DNA (ctDNA), Liquid biopsy, Non-small cell lung cancer (NSCLC)

\footnotetext{
* Correspondence: john.palma@roche.com

'Roche Sequencing Solutions, 4300 Hacienda Dr, Pleasanton \& Potsdam, California 94588, USA

Full list of author information is available at the end of the article
}

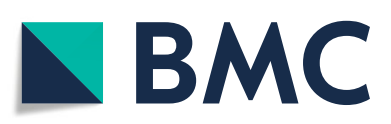

(- The Author(s). 2020 Open Access This article is licensed under a Creative Commons Attribution 4.0 International License, which permits use, sharing, adaptation, distribution and reproduction in any medium or format, as long as you give appropriate credit to the original author(s) and the source, provide a link to the Creative Commons licence, and indicate if changes were made. The images or other third party material in this article are included in the article's Creative Commons licence, unless indicated otherwise in a credit line to the material. If material is not included in the article's Creative Commons licence and your intended use is not permitted by statutory regulation or exceeds the permitted use, you will need to obtain permission directly from the copyright holder. To view a copy of this licence, visit http://creativecommons.org/licenses/by/4.0/ The Creative Commons Public Domain Dedication waiver (http://creativecommons.org/publicdomain/zero/1.0/) applies to the data made available in this article, unless otherwise stated in a credit line to the data. 


\section{Background}

Lung cancer is the leading cause of cancer death [1]. Non-small cell lung cancer (NSCLC) is the most common type of lung cancer, comprising $80-85 \%$ of all lung cancers [2]. About $40 \%$ of lung cancers were diagnosed at advanced stage [3]. The 5-year relative survival rate of stage IV NSCLC is only 6\% [2]. Over the past 20 years, treatments for advanced stage NSCLC have evolved from empirical cytotoxic therapies to more precision treatments including targeted therapies and immunotherapies. Therapies targeting specific genomic alterations, such as epidermal growth factor receptor (EGFR) mutations, anaplastic lymphoma kinase (ALK), ROS proto-oncogene 1 , receptor tyrosine kinase (ROS1) fusions, have greatly improved NSCLC management [4]. Assessing therapy response and monitoring disease progression are critical components of providing the right therapy at the right time and further improving overall survival.

Liquid biopsies have emerged as a crucial tool in cancer management. When tumor cells undergo apoptosis or necrosis, DNA is shed into the bloodstream $[5,6]$. This shed DNA (circulating tumor DNA [ctDNA]) contains gene mutations, representative of those found in primary and metastatic tumors [7-9]. The specific molecular changes identified in ctDNA could have diagnostic value and be used to predict therapy response and patient survival $[10,11]$. The ctDNA can be harvested via blood collection, and due to the relative ease with which "liquid biopsies" are performed, it is possible to obtain serial samples and to analyze changes in tumor composition, and to monitor treatment response and disease progression over time $[12,13]$. Moreover, because both primary and metastatic tumors shed DNA into the circulation, liquid biopsies are likely to provide a more comprehensive picture of the total cancer genome than traditional biopsies. As a consequence, the ability of ctDNA to assist in the diagnosis, treatment, and surveillance/monitoring of cancer has become an area of active research [14, 15].

Theoretically, liquid biopsies coupled with nextgeneration sequencing (NGS) can be used to monitor the evolution of NSCLC over time. If known resistance mutations emerge, the appropriateness of a prescribed treatment regimen could be reassessed [16]. The present analysis examined the ability of NGS coupled with ctDNA from serially acquired plasma samples to assess treatment response and/or disease progression and to explore the potential of ctDNA as a prognostic factor in subjects receiving first-line treatment for advanced NSCLC.

\section{Methods}

\section{Study design and subjects}

This was a retrospective sub-study of adults aged $\geq 18$ years participating in the prospective German Time
Series of Biomarkers in Lung Cancer (ZRLK) study. Study participants had not previously been treated systemically for advanced NSCLC, which was diagnosed per the Prevention, Diagnosis, Therapy, and Follow-up of Lung Cancer Interdisciplinary Guidelines from the German Respiratory Society and the German Cancer Society [17]. To be eligible for the sub-study, subjects had to have had histologically proven adenocarcinoma of the lung and at least 2 blood draws during first-line treatment (i.e., prior to clinical disease progression). All subjects provided written informed consent within the context of a prospective observational study, and the study adhered to Good Clinical Practice guidelines and Declaration of Helsinki principles.

Per study protocol, blood samples were collected at baseline, prior to each chemotherapy cycle, and about every 3 months during treatment-free intervals. Twenty $\mathrm{mL}$ of peripheral blood was collected in EDTA-coated tubes. Plasma was separated by centrifugation at $1500 \mathrm{x}$ $\mathrm{g}$ for $15 \mathrm{~min}$ within $2 \mathrm{~h}$ of sample collection and stored at $-80{ }^{\circ} \mathrm{C}$ until DNA extraction. In total, 445 blood samples from the 71 patient cohort were collected, with minimum 2 and maximum 18 samples per patient. The median number of blood samples per patient was 6 . Out of 71 subjects, $47(66.2 \%)$ had a pre-treatment tissue sample available and 42 of the $47(89.4 \%)$ had somatic variants detected. Distribution of baseline characteristics were compared between subjects with tissue biopsies ( $n=47)$ and the remaining subjects $(n=24)$ by Wilcoxon rank-sum test, Pearson's chi-square test, and Fisher's exact test as appropriate.

Cell-free DNA (cfDNA) was extracted from $4 \mathrm{~mL}$ of plasma, and tumor DNA was isolated from formalinfixed paraffin-embedded (FFPE) tumor tissue sections using the AVENIO cfDNA isolation kit (Roche, Branchburg, New Jersey) and KAPA Express Extract kit (Roche, Cape Town, South Africa), respectively. DNA yields were quantified by a Qubit fluorometer (ThermoFisher Scientific, Waltham, Massachusetts). The cfDNA yield had a range of 13 to $1164 \mathrm{ng}$ with the median of $58 \mathrm{ng}$. The amount of isolated tumor tissue DNA was from 63 ng to $15,420 \mathrm{ng}$ with a median of $1180 \mathrm{ng}$. cfDNA was sequenced using the AVENIO ctDNA Surveillance assay (Research Use Only; Roche, Branchburg, New Jersey), and tumor tissue DNA was sequenced using a FFPET assay (AVENIO Tumor Tissue Surveillance Kit, Research Use Only; in development; Roche, Branchburg, New Jersey). The median input mass for FFPE samples was $50 \mathrm{ng}$ (range 9-200 ng) and median input mass for plasma samples was $20 \mathrm{ng}$ (range 10-50 ng). Highthroughput sequencing was performed on the Illumina NextSeq 500 instrument (Illumina, San Diego, California). Both the AVENIO ctDNA Surveillance Kit and the prototype of AVENIO tumor tissue assays, which are 
based on Cancer Personalized Profiling by Deep Sequencing (CAPP-Seq) technology [15], utilize molecular barcoding and a hybrid-capture methodology to interrogate selected regions and recurrent mutations from 197 genes commonly mutated in cancer, including 17 important cancer-related genes (e.g., EGFR, KRAS, BRAF, TP53). The average de-duplicated sequencing depth of ctDNA and FFPE tumor tissue DNA was 2779 and 1116, respectively. Somatic variants were identified using the AVENIO ctDNA Analysis Software (Roche, Branchburg, New Jersey), which incorporates integrated digital error suppression [18] to remove polymerase chain reaction duplicates and stereotypical errors.

\section{Identification of variants in plasma-derived ctDNA and FFPE tumor tissue DNA}

Somatic variants in cfDNA were derived by filtering out predicted germline variants using an algorithm that is able to detect putative somatic variants with allele frequencies $<30 \%$ (not excluding whitelist mutation); this is possible because the algorithm relies on relevant information from multiple somatic mutations rather than on an allele frequency cut-off (described below). After using molecular barcoding and background polishing algorithms (adapted from those described in Newman et al., 2016) [19], the AVENIO ctDNA Surveillance Kit adaptively sets the lower threshold for variant calling based both on the background of a sample and error profile at a given position; no global lower threshold for allele frequencies is applied. Somatic variants in FFPE tumor tissue DNA were derived by post-call filters according to the following criteria: (1) variants had to be in nonrepetitive regions of the genome, (2) variants are not predicted to be germline variants (described below), (3) the population frequencies of variants had to be $<1 \%$ in the Exome Aggregation Consortium (ExAC) database, and (4) the allele frequencies of variants had to be $>5 \%$ (or $>3 \%$ if variants were well known hot-spot mutations). Both synonymous and non-synonymous somatic variants were selected for further analysis.

\section{Germline-variant filtering for each subject}

To accurately identify somatic mutations in the absence of matched normal sequences, a machine-learning algorithm (CSMutan) was developed that leveraged mutation databases (ExAC, Single Nucleotide Polymorphism database, 1000 Genomes Project, Catalogue of Somatic Mutations in Cancer, Cancer Genome Atlas) and variant allele frequencies. Using in-house sequencing data of plasma samples whose germline single nucleotide polymorphisms have been identified by comparison with matched normal DNA, we built a classifier to distinguish germline variants from somatic variants with a set of features derived from databases and samples' variants allele frequency. The detail description of the machine learning algorithm could be found in filed patent application [20]. This classifier was then used to predict germline variant profiles for individual subjects.

\section{ctDNA detection method}

The presence (ctDNA - positive) vs absence (ctDNAnegative) of mutant ctDNA was evaluated using a previously described Monte Carlo method [15] indicating whether a given set of variants for each sample was significantly higher than background across the sequenced genomic regions. For a given plasma sample, we started with a set of variants (n), which were high-confidence somatic mutations identified in the matched baseline tissue sample. Using Monte Carlo simulation, we compared the mean allele frequency of the reports against the null distribution of background allele alterations, which most are background sequencing errors, in the plasma sample. In the simulation, we conducted 10,000 iterations, each of which randomly picked $\mathrm{n}$ background allele alterations and calculated their mean allele frequency. The empirical $p$-value of the plasma sample was determined as percentile of mean allele frequency regarding to the null distribution of the background allele alterations. Plasma samples, whose variants with empirical $p$ values from the Monte Carlo method were less than 0.01 , were considered to be ctDNA-positive and otherwise ctDNA-negative.

\section{Correlation of allele frequencies to clinical response}

Subjects underwent routine CT imaging at baseline and after every two therapy cycles (not all data available); if clinically indicated, the frequency of CT imaging was adjusted. CT scans were evaluated by a reference radiologist at the Thoraxklinik, Ruprecht-Karls-University at Heidelberg according to Response Evaluation Criteria in Solid Tumors (RECIST) v1.1 [21]. The relationship between total ctDNA level or the presence/absence of ctDNA mutations and radiological response to treatment was assessed. Total ctDNA level was quantified by summing the allele frequency of each variant detected and normalizing to the total amount of input cfDNA. Progression-free survival (PFS) time was computed as difference in days between date of histological diagnosis and date of diagnosis of a progression of disease event defined by RECIST criteria or date of death of any course. The PFS time was censored for all other subjects at the date of the last imaging assessment. PFS was analyzed descriptively using the Kaplan-Meier method. Individual Cox proportional hazards models were used to assess the significance of each potential adjustment factor (age, sex, ECOG (0 vs. 1 or 2), smoking status (current smoker vs. never smoker vs. quit smoking), and 
disease stage (IIIA vs. IIIB vs. IV) with PFS. None of these factors were significantly associated with PFS. Two final unadjusted Cox proportional hazard regression models were used to calculate hazard ratios (HRs) with 95\% confidence intervals (CIs) for ctDNA mutation status for the first available post-treatment plasma sample and the last available post-treatment plasma sample. Molecular progression was defined as mutational allele frequencies with consecutive increases from baseline.

\section{Results}

In total, 71 subjects with NSCLC eligible for first-line treatment were enrolled between February 2014 and June 2016. The baseline characteristics of these subjects are summarized in Table 1. The average age was 62.5 years, and the majority of subjects were male $(63.4 \%)$ and had adenocarcinoma (96\%). In total, 5.6, 11.3, and $83.1 \%$ of subjects presented with stage IIIA, IIIB, and IV disease, respectively. Cisplatin/carboplatin plus pemetrexed were the chemotherapy regimen for the majority of subjects. Some patients also

Table 1 Baseline Demographics and Disease Characteristics

\begin{tabular}{|c|c|c|c|c|}
\hline & Total Study Cohort $(\boldsymbol{n}=71)$ & $\begin{array}{l}\text { Subset of Subjects with } \\
\text { Pretreatment Tissue } \\
\text { Biopsies }(n=47)\end{array}$ & Remaining Subjects $(n=24)$ & $\boldsymbol{P}$-value \\
\hline Age (years) & & & & 0.9466 \\
\hline Mean (SD) & $62.54(10.0)$ & $62.43(9.2)$ & $62.75(11.6)$ & \\
\hline Median & 62 & 62 & 61.5 & \\
\hline Age, $n(\%)$ & & & & 0.8108 \\
\hline$<70$ & $52(73)$ & $34(72)$ & $18(75)$ & \\
\hline$>=70$ & $19(27)$ & $13(28)$ & $6(25)$ & \\
\hline Sex, $n(\%)$ & & & & 0.5282 \\
\hline Female & $26(37)$ & $16(34)$ & $10(42)$ & \\
\hline Male & $45(63)$ & $31(66)$ & $14(58)$ & \\
\hline ECOG Status, n(\%) & & & & 0.1672 \\
\hline 0 & $22(31)$ & $17(36)$ & $5(21)$ & \\
\hline 1 & $44(62)$ & $28(60)$ & $16(67)$ & \\
\hline 2 & $3(4)$ & $2(4)$ & $1(4)$ & \\
\hline 3 & $2(3)$ & - & $2(8)$ & \\
\hline Stage, n(\%) & & & & 0.3136 \\
\hline IIIA & $4(6)$ & $2(4)$ & $2(8)$ & \\
\hline$\| \mathrm{B}$ & $8(11)$ & $7(15)$ & $1(4)$ & \\
\hline IV & $59(83)$ & $38(81)$ & $21(88)$ & \\
\hline Histology, n(\%) & & & & 0.4137 \\
\hline Adenocarcinoma & $68(96)$ & $45(96)$ & $23(96)$ & \\
\hline Adenocarcinoma|Large cell carcinoma ${ }^{a}$ & $1(1)$ & - & $1(4)$ & \\
\hline Adenocarcinoma|Squamous cell carcinoma ${ }^{a}$ & $2(3)$ & $2(4)$ & - & \\
\hline $1 \mathrm{~L}$ Therapy Received, $\mathrm{n}(\%)$ & & & & 0.8582 \\
\hline Chemotherapy Only ${ }^{\mathrm{b}}$ & $31(44)$ & $21(45)$ & $10(42)$ & \\
\hline Chemo+Radiation $^{c}$ & $25(35)$ & $17(36)$ & $8(33)$ & \\
\hline Chemo+Targeted $^{d}$ & $9(13)$ & $6(13)$ & $3(13)$ & \\
\hline Chemo+Radiation+Targeted $^{d}$ & $6(8)$ & $3(6)$ & $3(13)$ & \\
\hline Smoking Status, n(\%) & & & & 0.8495 \\
\hline Never Smoker & $11(15)$ & $6(13)$ & $5(21)$ & \\
\hline Quit more than 5 years ago & $9(13)$ & $6(13)$ & $3(13)$ & \\
\hline Quit less than 5 years ago & $9(13)$ & $6(13)$ & $3(13)$ & \\
\hline Current Smoker & $42(59)$ & $29(62)$ & $13(54)$ & \\
\hline
\end{tabular}

ECOG, Eastern Cooperative Oncology Group; SD, standard deviation. ${ }^{a}$ All subjects received an initial diagnosis of adenocarcinoma; however, additional histology was reported after re-biopsy; ${ }^{b}$ Cisplatin/carboplatin plus pemetrexed; ${ }^{c}$ Radiation included for brain or bone metastatic sites; ${ }^{d}$ Targeted therapy was bevacizumab in 13 subjects, erlotinib plus chemotherapy and radiation in 1 subject, and 1 subject received Nivolumab after chemotherapy and bevacizumab 
received Bevacizumab in addition to chemo (Supplementary Table 1). From these 71 subjects, 445 longitudinal plasma samples (range of 2-18 samples per subject) were collected over an average of 5 months (range, 1-25). Fortyseven of the 71 subjects had baseline tissue sample available. Baseline characteristics were not significantly different between subjects with tissue biopsies $(n=47)$ compared to the remaining subjects $(n=24)$, suggesting that the 47 subjects did not differ from the total study cohort. (Table 1).

Somatic variations were detected in $91.5 \%(407 / 445)$ of plasma samples, with an average of five mutations per sample. Of the 47 subjects with available tissue biopsies, somatic mutations were detected in 42 (89.4\%), with TP53, $K R A S$, and KEAP1 being the most frequently mutated genes (Table 2). The average number of mutations detected in tissue samples was six. The mean allele frequency for mutations detected in plasma-derived ctDNA was $1.74 \%$, ranging from 0.03 to $45.28 \%$; the corresponding value in FFPE tumor tissue DNA was $21.7 \%$, ranging from 3.29 to $86.82 \%$. The most frequent mutations in all ctDNA samples were in TP53 (27.9\%), KRAS (21.3\%), and NPAP1 (16.4\%) (Supplementary Table 2). In baseline ctDNA samples, the top mutated genes were TP53 (43.1\%), KRAS (29.4\%), NPAP1 (29.4\%), LRRTM4 (17.6\%), CSMD3 (15.7\%), and MET (15.7\%) (Supplementary Table 3). On average75.2\% of somatic variants identified in tissue were also identified in the matched baseline ctDNA plasma sample.

By utilizing both the AVENIO ctDNA Surveillance Kit and the tumor tissue Surveillance kit, we were able to track tumor mutation changes over time. In the two patient examples forthwith with corresponding imaging data, detection of ctDNA preceded radiographic progression (per RECIST 1.1). In some subjects, the allele frequencies of mutations detected in ctDNA increased consecutively 3-5 months before clinical evidence of disease progression. For example, in one subject who had been treated with carboplatin plus pemetrexed, the allele frequency of mutations in TP53 (CDS mutation: c.712 T > G) increased from 3.0\% at diagnosis to $8.5 \%$ at day 47 and to $10.6 \%$ at day 68 posttreatment. However, the CT scans did not show evidence of disease progression $(75 \%$ increase in the diameters of

Table 2 Gene Mutation Frequencies in Pre-treatment Tissue Biopsies

\begin{tabular}{ll}
\hline Gene & Subset of Subjects with Pre-Treatment Tissue Biopsies $(\mathrm{N}=47)$ \\
\hline TP53 & $20(42.6)$ \\
KRAS & $12(25.5)$ \\
KEAP1 & $9(19.1)$ \\
BRAF & $6(12.8)$ \\
STK11 & $5(10.6)$ \\
ERBB2 & $5(10.6)$ \\
EGFR & $2(4.2)$ \\
\hline
\end{tabular}

Data are $\mathrm{n}(\%)$ target lesions plus new lesion development in the liver) until day 159-a difference of 91 days (Fig. 1a). Similar trends were uncovered in another subject in whom mutations in TP53 and SLITRK5 were detected. Molecular progression (defined as a consecutive increase in mutation allele frequencies) was apparent on day 33 postdiagnosis of metastatic disease, but clinical disease progression was not seen until day 174 (Fig. 1b). We also examined the relationship between total ctDNA level, which sum up all mutant molecules detected in plasma sample, and treatment response. For example, the analysis had shown that the ctDNA level from one study subject changed with therapy administration and clinical disease progression (specifically, development of a new non-target lesion) (Fig. 1c).

The prognostic value of cancer mutations in plasmaderived ctDNA was further evaluated. When stratifying subjects by ctDNA mutation status in the first available post-treatment plasma sample, we found that ctDNApositive subjects vs. ctDNA-negative subjects were associated with reduced PFS. Median PFS was 5.6 months in the ctDNA-positive group and 8.9 months in the ctDNA-negative group. The HR for the comparison of the two groups (ctDNA-positive vs. ctDNA-negative) was 2.3 (95\% CI, 1.1-4.8; log-rank $p=0.0183$ ) (Fig. 2a). A sensitivity analysis in which ctDNA mutation status was determined using the last available plasma sample yielded similar results (Fig. 2b). Analysis using individual Cox proportional hazards models had demonstrated that age, gender, ECOG status, smoking status, and disease stage were not significantly associated with disease progression, as shown in Table 3.

\section{Discussion}

In this study, longitudinal monitoring analysis of somatic mutations in plasma samples from subjects receiving first-line treatment for advanced adenocarcinoma of the lung was explored. The average number of mutations detected in tissue and plasma was very similar, and about $90 \%$ of all tumor tissue and plasma sample tested had 1 or more variants identified. In the cohort of this study subjects, the most frequently detected mutations in FFPE tumor tissue DNA were TP53, KRAS, and $K E A P 1$, which is consistent with the findings of Chaudhuri et al. [22] The prevalence of EGFR mutation in this study (4.2\%) was lower than that of TCGA data [18]. This may be due to the small tissue sample size and the nature of this prospective collection that part of the EGFR mutated population were enrolled in other clinical studies and tissue samples were not available for this analysis.

Serial plasma sampling provides an opportunity to detect mutations emerging over time and to monitor disease progression based on the presence or change of 




B.


Fig. 1 Correlation between allele frequencies in ctDNA and clinical disease in a subject with $\mathbf{a}$ a single somatic mutation and $\mathbf{b}$ two somatic mutations. c Correlation between total ctDNA load and clinical disease. The duration of treatment with chemotherapy is represented by the green band, radiation by the beige band, and targeted therapy by the light purple band. The overlap in radiation and targeted therapy is represented by the dark purple band. ctDNA, circulating tumor DNA; mGE, mutated genome equivalent; PD, progressive disease 


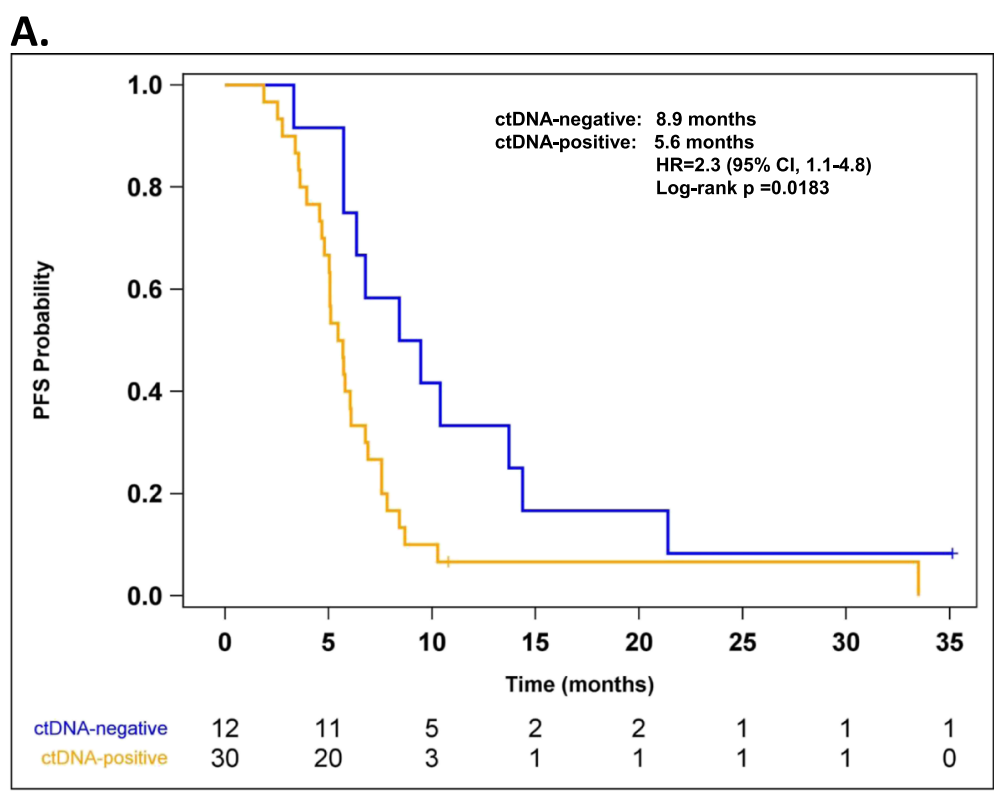

B.



Fig. 2 Median PFS in subjects stratified by the presence/absence of mutations in ctDNA isolated from the a first available post-treatment plasma sample and $\mathbf{b}$ last available post-treatment plasma sample. $\mathrm{Cl}$, confidence interval; ctDNA, circulating tumor DNA; HR, hazard ratio; PFS, progression-free survival

mutant molecules. Moreover, liquid biopsies contain the genomes of primary and metastatic tumors [14]. This contrasts with traditional biopsies, which excise tissue from only a single tumor location and which are thus subject to tumor and genomic heterogeneity. Although ctDNA levels in blood can be low, the sequencing of multiple plasma samples-each of which potentially contains the genomes of multiple metastatic tumorsincreases the likelihood of detecting somatic, diseaseassociated mutations, including low-frequency variants.
Through use of the AVENIO ctDNA Surveillance Kit (Research Use Only), which enabled us to assess changes in the mutation status of both single and multiple cancer genes over time, we found the presence of ctDNA in post-treatment plasma samples to be a risk factor for disease progression, independent of clinical parameters. Moreover, molecular relapse in 2 patient cases was shown to precede clinical relapse by $3-5$ months, a finding supported by the work of others [15, 22-24]. For example, Phallen J et al [23] have 
Table 3 Association of Potential Adjustment Factors with Progression Free Survival

\begin{tabular}{|c|c|c|c|}
\hline \multirow[b]{2}{*}{ Variable } & \multirow[b]{2}{*}{ Level } & \multicolumn{2}{|l|}{ Unadjusted } \\
\hline & & HR (95\% Cl) & $\mathbf{P}$ \\
\hline Age & & $0.99(0.96,1.03)$ & 0.6628 \\
\hline Sex & Female (Reference $=$ Male) & $1.24(0.63,2.46)$ & 0.5350 \\
\hline ECOG & 0 (Reference $=1$ or 2 ) & $1.22(0.63,2.37)$ & 0.5554 \\
\hline \multirow[t]{2}{*}{ Smoking Status } & Current Smoker (Reference $=$ Quit Smoking) & $0.98(0.48,2.00)$ & 0.2816 \\
\hline & Never Smoker (Reference = Quit Smoking) & $0.37(0.10,1.37)$ & \\
\hline \multirow[t]{2}{*}{ Disease Stage } & IIIA (Reference = IV) & $1.07(0.25,4.54)$ & 0.7842 \\
\hline & IIIB (Reference = IV) & $1.38(0.56,3.37)$ & \\
\hline
\end{tabular}

Individual unadjusted Cox proportional hazards models were used for each potential adjustment factor

Global $p$-values presented for categorical variables with more than 2 levels

demonstrated that ctDNA non-responders had a significantly shorter median progression-free survival compared to ctDNA responders in the metastatic NSCLC population treated with targeted tyrosine kinase inhibitors. A recent study in KRAS mutated NSCLC patients had also shown that KRAS mutation presence or dynamic change in post treatment plasma was significantly associated with increased probability of experiencing progressive disease, and PFS and OS [25]. Therefore, changing therapy when ctDNA level is increasing, before radiographic progression, might potentially improve the clinical outcome. Clinical studies are needed to explore this potential.

We also found total ctDNA level to be prognostic of disease progression and ctDNA-positivity vs ctDNAnegativity to be associated with reduced median PFS. Collectively, these preliminary results suggest that deep sequencing of serially acquired liquid biopsies from subjects with advanced NSCLC has the potential to facilitate longitudinal disease monitoring and to predict disease progression earlier than radiologic scans. The identification of resistance clones or signatures prior to clinical disease progression may provide an opportunity to transition sooner to alternative therapy, but additional studies are needed to validate this concept, and prospective clinical trials are necessary to demonstrate its clinical utility.

\section{Conclusions}

The presence of ctDNA during treatment of late stage NSCLC is a significant risk factor for survival. Total ctDNA level may reflect disease burden at a specific time point. ctDNA increase could be detected months before clinical progression, and this information could potentially be used for disease monitoring in conjunction with imaging which might have a positive impact on patient outcome. ctDNA assessment may provide significant value to improve therapy selection, disease surveillance and monitoring, and drug resistant mutation detection.

\section{Supplementary information}

Supplementary information accompanies this paper at https://doi.org/10. 1186/s12885-020-07340-z.

Additional file 1: Supplementary Table 1. Treatment regimens of study subjects received.

Additional file 2: Supplementary Table 2. Somatic variants detected in all plasma samples.

Additional file 3: Supplementary Table 3. Somatic variants detected in baseline plasma samples.

\section{Abbreviations}

CAPP-Seq: Cancer Personalized Profiling by Deep Sequencing; cfDNA: Cellfree DNA; Cl: Confidence interval; ctDNA: circulating tumor DNA;

ExAC: Exome Aggregation Consortium; FFPE: Formalin-fixed paraffinembedded; HR: Hazard ratio; NGS: Next-generation sequencing; NSCLC: Nonsmall cell lung cancer; PFS: Progression-free survival; RECIST: Response Evaluation Criteria in Solid Tumors; ZRLK: German Time Series of Biomarkers in Lung Cancer

\section{Acknowledgements}

The authors wish to thank the following individuals from Roche Sequencing Solutions for their assistance: Preeti Lal, Nasiema Wingate-Pearse, David Zhang, Alexander F. Lovejoy, Daniel M. Klass, Janet Jin, Frederike Fuhlbrück, Diana Köpke, and Kati Probst. Support for third-party writing assistance for this manuscript was provided by Tiffany DeSimone, PhD, of CodonMedical, an Ashfield Company, part of UDG Healthcare plc, and was funded by F. Hoffmann-La Roche.

\section{Authors' contributions}

JJ, HPA, SJY, LY, AB, NT, CJ, and JP contributed to protocol development, data analysis, and drafted the manuscript. JJ, HPA, HJA, RK, and JP contributed to conceived of the study and participated its coordination. HPA, ML, SS, MF, SM, SF, and NT contributed to data check and information retrieval. JJ researched the literature. JJ, HAP, and JP designed the study and contributed to overall management of the study. All authors reviewed the manuscript and approved the final version of the manuscript.

\section{Funding}

F. Hoffmann-La Roche Ltd. supported this study and was involved in the design of the study; collection, analysis, and interpretation of the data; writing of the report; and the decision to submit the article for publication. Support for third-party writing assistance for this manuscript was provided by Tiffany DeSimone, PhD, of CodonMedical, an Ashfield Company, part of UDG Healthcare plc, and was funded by F. Hoffmann-La Roche. 


\section{Availability of data and materials}

The datasets generated and/or analysed during the current study are not publicly available due to General Data Protection Regulation and sensitive genetic information.

\section{Ethics approval and consent to participate}

The leading ethics committee of the state's association of physicians of the principal investigator, Dr. Rainer Krügel, practicing medicine in the state of Brandenburg, has voted that the study does not violate any ethical consideration, and the ethics committees, namely Landesärztekammer Brandenburg, of physicians associations of German states, namely the free state Saxony, the free state Thuringia, and the state of Saxony-Anhalt and the state of Brandenburg, have endorsed and granted the approval of the study protocol, which is sponsored by Signature Diagnostics $\mathrm{GmbH}$, a 100\% subsidiary of Roche Deutschland GmbH (Reference number S 26(a)/2013). All patients provided written informed consent to participate in this study.

\section{Consent for publication}

Not applicable.

\section{Competing interests}

John Jiang, Hans-Peter Adams, Maria Lange, Sandra Siemann, Mirjam Feldkamp, Sylvie McNamara, Stephanie J. Yaung, Lijing Yao, Aarthi Balasubramanyam, Nalin Tikoo, Christine Ju, and John F. Palma are salaried employees of F. Hoffmann-La Roche. Sebastian Froehler is a salaried employee of F. Hoffmann-La Roche, Basel, Switzerland. H. Jost Achenbach reports personal fees from AstraZeneca, Bristol-Myers Squibb, Boehringer Ingelheim, Novartis, Insmed, Grifols and Roche outside the submitted work. Rainer Krügel reports personal fees from Bristol-Myers Squibb, Boehringer Ingelheim, Novartis, Merck Sharp \& Dohme, Mediolanum, and Roche outside the submitted work.

\section{Author details}

'Roche Sequencing Solutions, 4300 Hacienda Dr, Pleasanton \& Potsdam, California 94588, USA. ${ }^{2}$ Signature Diagnostics GmbH, Hermannswerder 20A, 14473 Potsdam, Germany. ${ }^{3}$ Roche Molecular Systems, 4300 Hacienda Dr., Pleasanton, CA 94588, USA. ${ }^{4}$ Lungenklinik Lostau, Lindenstraße 2, 39291 Lostau, Germany. ${ }^{5}$ Johanniter Krankenhaus im Fläming, Johanniterstraße 1, 14929 Treuenbrietzen, Germany.

\section{Received: 3 December 2019 Accepted: 25 August 2020}

Published online: 15 September 2020

\section{References}

1. Siegel RL, Miller KD, Jemal A. Cancer statistics. CA Cancer J Clin. 2018;68(1): 7-30.

2. American Cancer Society. What is non-small cell lung cancer? https://www. cancer.org/cancer/non-small-cell-lung-cancer/about/what-is-non-small-celllung-cancer.html. Accessed January 11, 2018.

3. Morgensztern D, Ng SH, Gao F, Govindan R. Trends in stage distribution for subjects with non-small cell lung cancer: a National Cancer Database survey. J Thorac Oncol. 2010;5(1):29-33.

4. Jordan EJ, Kim HR, Arcila ME, Barron D, Chakravarty D, Gao J, et al. Prospective comprehensive molecular characterization of lung adenocarcinomas for efficient patient matching to approved and emerging therapies. Cancer Discov. 2017;7(6):596-609.

5. Stroun M, Lyautey J, Lederrey C, Olson-Sand A, Anker P. About the possible origin and mechanism of circulating DNA apoptosis and active DNA release. Clin Chim Acta. 2001;313(1-2):139-42.

6. Jahr S, Hentze H, Englisch S, Hardt D, Fackelmayer FO, Hesch RD, Knippers R. DNA fragments in the blood plasma of cancer subjects: quantitations and evidence for their origin from apoptotic and necrotic cells. Cancer Res. 2001;61(4):1659-65.

7. Schwarzenbach $\mathrm{H}$, Hoon DS, Pantel K. Cell-free nucleic acids as biomarkers in cancer subjects. Nat Rev Cancer. 2011;11(6):426-37.

8. Taniguchi K, Uchida J, Nishino K, Kumagai T, Okuyama T, Okami J, et al. Quantitative detection of EGFR mutations in circulating tumor DNA derived from lung adenocarcinomas. Clin Cancer Res. 2011;17(24):7808-15.

9. Bettegowda C, Sausen M, Leary RJ, Kinde I, Wang Y, Agrawal N, et al. Detection of Circulating Tumor DNA in Early- and Late-Stage Human Malignancies. Sci Transl Med. 2014;6(224):224ra224.
10. Ma M, Zhu H, Zhang C, Sun X, Gao X, Chen G. "Liquid biopsy" - ctDNA detection with great potential and challenges. Ann Transl Med. 2015;3(16): 235.

11. Kimura T, Holland WS, Kawaguchi T, Williamson SK, Chansky K, Crowley JJ, et al. Mutant DNA in plasma of lung cancer subjects: potential for monitoring response to therapy. Ann N Y Acad Sci. 1022:55-60.

12. Lee JY, Qing X, Xiumin W, Yali B, Chi S, Bak SH, Lee HY, Sun JM, Lee SH, Ahn JS, Cho EK, Kim DW, Kim HR, Min YJ, Jung SH, Park K, Mao M, Ahn MJ. Longitudinal monitoring of EGFR mutations in plasma predicts outcomes of NSCLC subjects treated with EGFR TKIs: Korean lung Cancer consortium (KLCC-12-02). Oncotarget. 2016;7(6):6984-93.

13. Almodovar K, lams WT, Meador CB, Zhao Z, York S, Horn L, Yan Y, Hernandez J, Chen H, Shyr Y, Lim LP, Raymond CK, Lovly CM. Longitudinal cell-free DNA analysis in subjects with small cell lung cancer reveals dynamic insights into treatment efficacy and disease relapse. J Thorac Oncol. 2017. https://doi.org/10.1016/j.jtho.2017.09.1951 [Epub ahead of print].

14. Diaz LA Jr, Bardelli A. Liquid biopsies: genotyping circulating tumor DNA. J Clin Oncol. 2014;32(6):579-86.

15. Newman AM, Bratman SV, To J, Wynne JF, Eclov NC, Modlin LA, Liu CL, Neal JW, Wakelee HA, Merritt RE, Shrager JB, Loo BW Jr, Alizadeh AA, Diehn M. An ultrasensitive method for quantitating circulating tumor DNA with broad patient coverage. Nat Med. 2014;20(5):548-54.

16. Suda K, Murakami I, Sakai K, Mizuuchi H, Shimizu S, Sato K, Tomizawa K, Tomida S, Yatabe Y, Nishio K, Mitsudomi T. Small cell lung cancer transformation and T790M mutation: complimentary roles in acquired resistance to kinase inhibitors in lung cancer. Sci Rep. 2015;5:14447.

17. Goeckenjan G, Sitter H, Thomas M, et al. Prävention, Diagnostik, Therapie und Nachsorge des Lungenkarzinoms [German]. Pneumologie. 2011;65:5175.

18. Newman AM, Lovejoy AF, Klass DM, Kurtz DM, Chabon JJ, Scherer F, Stehr H, Liu CL, Bratman SV, Say C, Zhou L, Carter JN, West RB, Sledge GW, Shrager JB, Loo BW Jr, Neal JW, Wakelee HA, Diehn M, Alizadeh AA. Integrated digital error suppression for improved detection of circulating tumor DNA. Nat Biotechnol. 2016;34:547-55.

19. Cancer Genome Atlas Research Network. Comprehensive molecular profiling of lung adenocarcinoma. Nature. 2014;511(7511):543-50. 22.

20. US patent filing WO 2019/016353 Al: Classifying somatic mutations from heterogenous sample. https://patentimages.storage.googleapis.com/ec/f6/4 0/b751c3e59fc69f/WO2019016353A1.pdf.

21. Eisenhauer EA, Therasse P, Bogaerts J, Schwartz LH, Sargent D, Ford R, Dancey J, Arbuck S, Gwyther S, Mooney M, Rubinstein L, Shankar L, Dodd L, Kaplan R, Lacombe D, Verweij J. New response evaluation criteria in solid tumours: revised RECIST guideline (version 1.1). Eur J Cancer. 2009;45(2): 228-47.

22. Chaudhuri AA, Chabon JJ, Lovejoy AF, Newman AM, Stehr H, Azad TD, et al. Early detection of molecular residual disease in localized lung cancer by circulating tumor DNA profiling. Cancer Discov. 2017;7(12):1394-403.

23. Phallen J, Leal A, Woodward BD, Forde PM, Naidoo J, Marrone KA, Brahmer $J R$, Fiksel J, et al. Early noninvasive detection of response to targeted therapy in non-small cell lung Cancer. Cancer Res. 2019;79(6):1204-13.

24. Abbosh C, Birkbak NJ, Wilson GA, Jamal-Hanjani M, Constantin T, Salari R, et al. Phylogenetic ctDNA analysis depicts early-stage lung cancer evolution. Nature. 2017;545(7655):446-51.

25. Zulato E, Attili I, Pavan A, Nardo G, Bianco PD, Bragadin AB, et al. Early assessment of KRAS mutation in cfDNA correlates with risk of progression and death in advanced non-small-cell lung cancer. Br J Cancer. 2020;123: 81-91.

\section{Publisher's Note}

Springer Nature remains neutral with regard to jurisdictional claims in published maps and institutional affiliations. 